\title{
Multiple USB Webcam Link Control System for Reading QR Codes of Micro PCR Biochip Calibration Factor Values
}

\author{
SeungCheol Lee ${ }^{1}$, Jong-Dae Kim ${ }^{2,3}$, Yu-Seop Kim ${ }^{2,3}$, Hye-Jeong Song ${ }^{2,3}$ and \\ Chan-Young Park ${ }^{2,3 *}$ \\ ${ }^{1}$ Department of Computer Engineering, Hallym University, Korea \\ ${ }^{2}$ Department of Ubiquitous Computing, Hallym University, Korea \\ ${ }^{3}$ Bio-IT Research Center, Hallym University, Korea \\ \{ leesc4957, kimjd, yskim01, hjsong, cypark\}@hallym.ac.kr
}

\begin{abstract}
Detection systems in a range of fields including medical science, food safety, and environmental monitoring use miniaturized polymerase chain reactions that have lab-ona-chip technologies. The chip used for a micro PCR needs the correction factor of the chip temperature sensor for the exact measurement and control of temperature. The correction factor of the temperature sensor is obtained from the chip, generated as a quick response code, and attached to the chip. A universal serial bus camera connected to a $P C$ reads the $Q R$ codes, and the data from the $Q R$ codes is used to correct the measured temperature. However, because several USB cameras cannot be operated on a single PC, users must bear the inconvenience of physically connecting USB cameras to PCs for use. In this thesis, we establish a system that automatically turns the USB cameras on and off by operating solid state relay modules and a micro controller unit used in micro PCSs. As a result, preparing micro PCRs for experiments has become more convenient and less time consuming.
\end{abstract}

Keywords: PCR, Lab-on-a-Chip, Bio Chip, quick response $(Q R)$ code, Solid State Relay

\section{Introduction}

Through evolution and development, today's humans are facing more chemical and biological threats, such as viral and bacterial contagion, than external physical risk factors. The most common current method of virus detection is molecular diagnostics, which detects the hexane of the sources of diseases, viruses and bacteria, to determine the disease source and contagion risk. The molecular diagnostic technique consists of four steps: sampling of body fluid; extracting genes from the sample; and using polymerase chain reaction (PCR) to amplify and analyze the genes

This technique can precisely diagnose infinitesimal amounts of pathogens because of its high degree of sensitivity and uniqueness derived from the gene amplification process. However, it has some drawbacks in that costly analysis equipment and reagents are needed for PCRs and electrophoresis, and that this technique must be conducted by skillful technicians. Furthermore, the analysis equipment takes up a large amount of space and analysis takes a long time.

The lab-on-a-chip technique has received much attention because it can overcome the disadvantages of conventional gene diagnostic techniques and can analyze genes on the spot.

A representative example of the lab-on-a-chip is microelectromechanical systems

${ }^{*}$ Corresponding Author 
(MEMS), a technology in which the whole process and analysis stages including the dilution, mixture, reaction, separation, and measurements of a sample, are carried out on a single chip [1].

A micro PCR through MEMS features a shorter analysis time than conventional PCRs and requires less sample usage, provides rapid heating and cooling rates, decreases power consumption for integrating various techniques and for operating equipment, and reduces cross-contamination and biochemical risks [2,3]. Although general PCRs can simultaneously conduct tens of DNA amplifications, the micro PCR processes one item at a time; so, to overcome this disadvantage, it is necessary to operate several units of micro PCRs simultaneously.

The chip for a micro PCR must have lower or no error rate between the measured temperature and actual temperature values. To that effect, it is necessary to obtain the correction factor value of a chip temperature sensor in order to correct the measured temperature value accordingly. The correction factor value obtained through the temperature sensor correction of each chip is sent to a PC that uses PCRs. When saving a correction factor value of the chip temperature sensor onto a chip to send it to a $\mathrm{PC}$, a quick response $(\mathrm{QR})$ code is used. A $\mathrm{QR}$ code is a two dimensional barcode that consists of a black and white grid pattern that contains information. It can save numbers and text data including alphabets and Chinese characters with a two-dimensional barcode of expanded format and content, which has overcome the capacity limitation of the barcode that had been widely used. Reading the QR code typically requires a digital camera or a scanner [4].

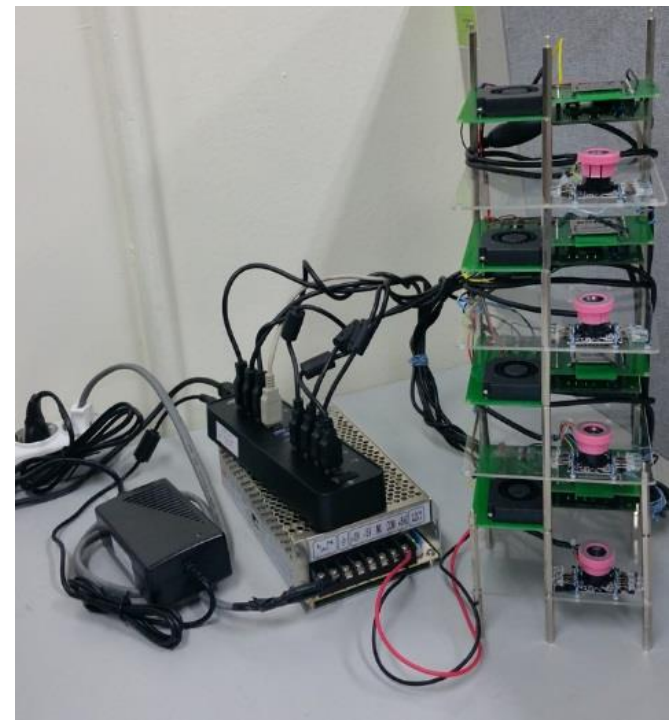

Figure 1. Micro PCR

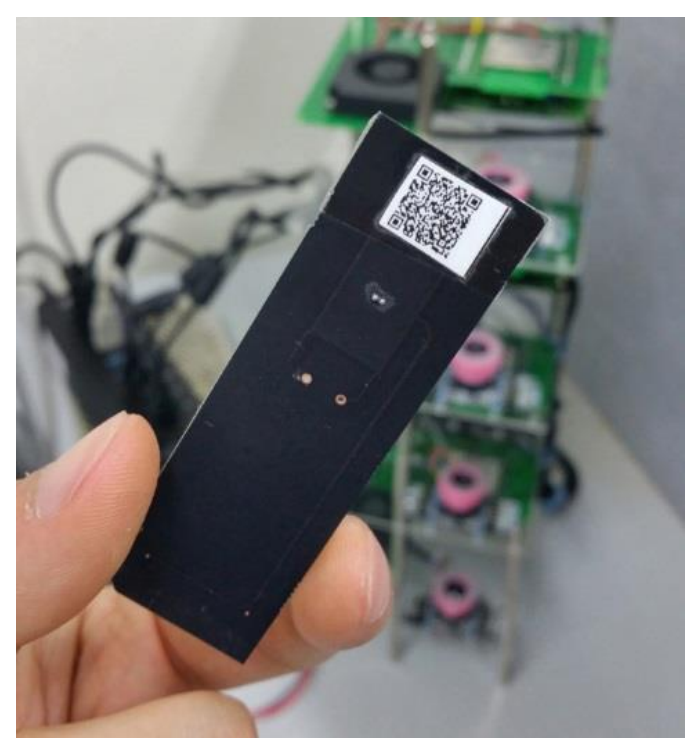

Figure 2. Biochip with a QR Code

Figure 1 shows the micro PCR that we use in this study. A total of four micro PCRs are each stacked on four racks, and a PC simultaneously operates the four micro PCRs. The micro PCRs must apply a correction factor value to each chip by reading different QR codes. For this task, USB cameras are installed on each micro PCR. Figure 2 shows, attached to the bottom of a biochip, a QR code containing a chip correction factor value. Once a biochip is mounted on a micro PCR, the USB camera installed on the micro PCR faces a QR code. When several cameras are operated on a PC, only the camera first connected to the PC functions, and the others do not. Using the other cameras requires disconnecting the link between the first-connected camera and the PC. This is inconvenient and delays test preparations because the user has to disconnect the link in person whenever a required camera will be in use. Therefore, we suggest a solution to this 
problem in camera operation by using a micro controller unit (MCU) and a solid-state relay (SSR).

\section{Hardware Design}

\subsection{USB Device VCC Cable}

The Universal Serial Bus (USB) is one of the input/output standards for connecting between computers and peripheral devices. Figure 3 shows a USB 2.0 cable. It consists of four pins: pin $1 \mathrm{VCC} 5 \mathrm{~V}$, pin 2 DATA-, pin $3 \mathrm{DATA}+$, and pin 4 ground (GND) [5].

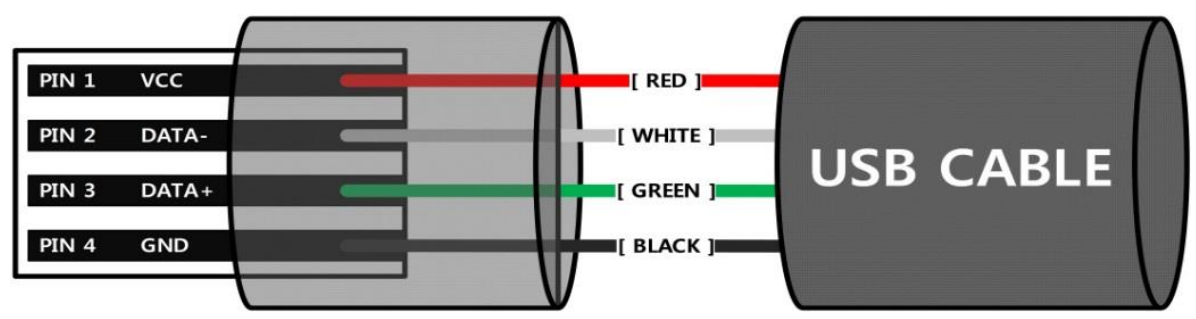

Figure 3. USB 2.0 Cable

When the VCC is blocked, USB devices cannot operate due to disconnected power. Even when the USB devices are physically connected to USB ports, a PC cannot recognize the USB devices because the devices do not work.

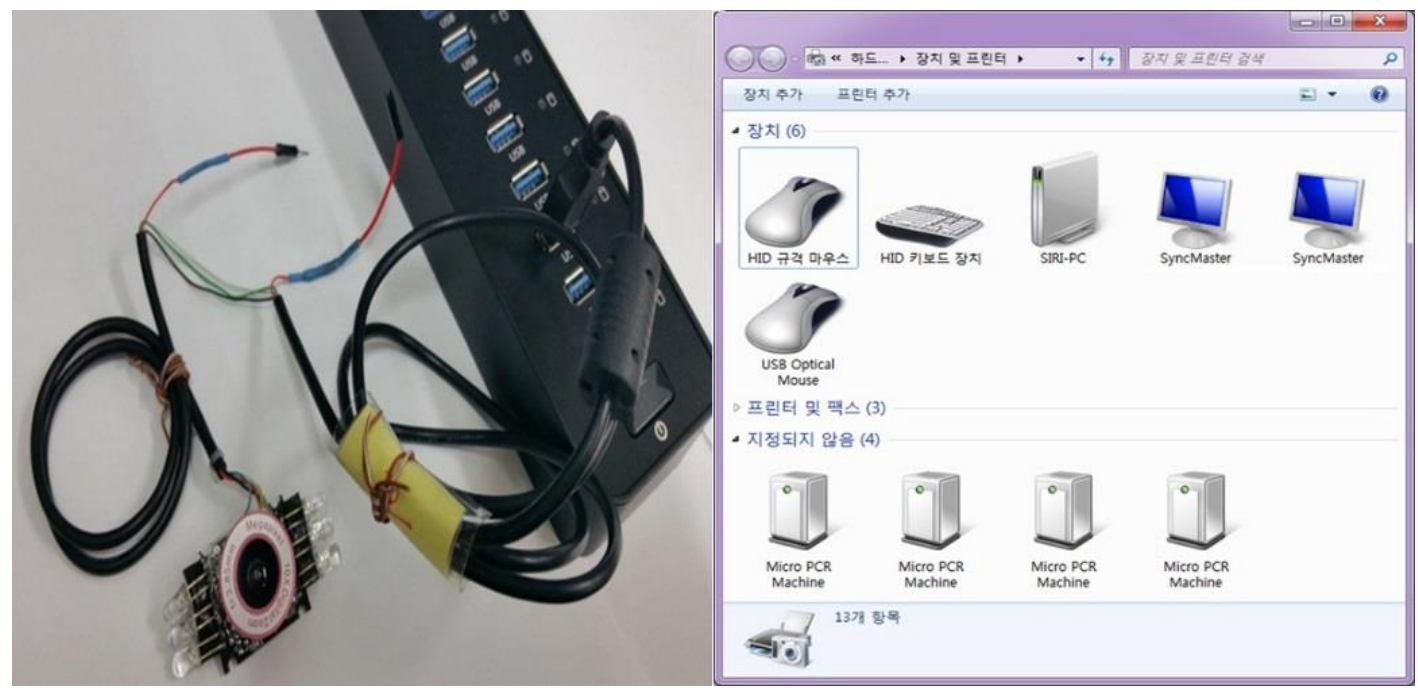

Figure 4. Disconnected USB Camera VCC Cable

Figure 4 shows images in which the PC recognizes the USB devices after disconnection of the jumper wires in charge of the VCC signals from the USB cables in the USB camera. As mentioned previously, a PC does not recognize USB devices because the VCC of the USB cables is blocked, even when the USB camera is connected to the USB ports of the PC. 


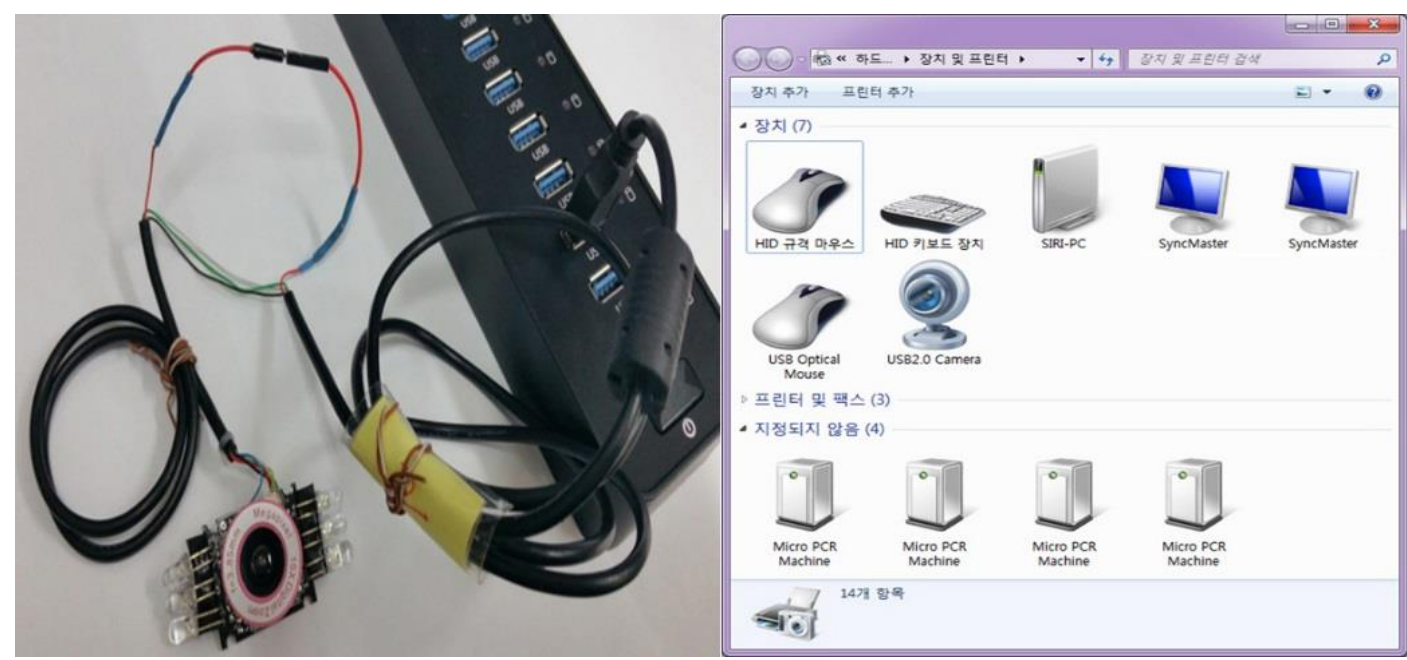

Figure 5. Reconnected USB Camera VCC Cable

Figure 5 shows images in which the PC recognizes the USB devices when the detached USB VCC jumper wires are reconnected. It can be confirmed that once the USB camera that is not operational due to the disconnection from the VCC is reconnected to the VCC, the camera becomes operational, and the PC recognizes the USB devices as well. USB cameras must be controlled by using the MCU and SSR based on such features of USB devices.

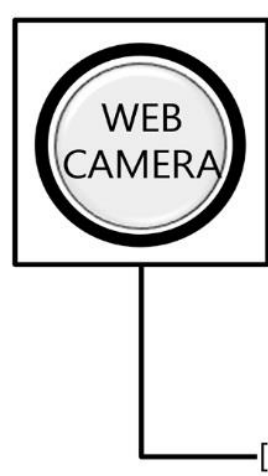

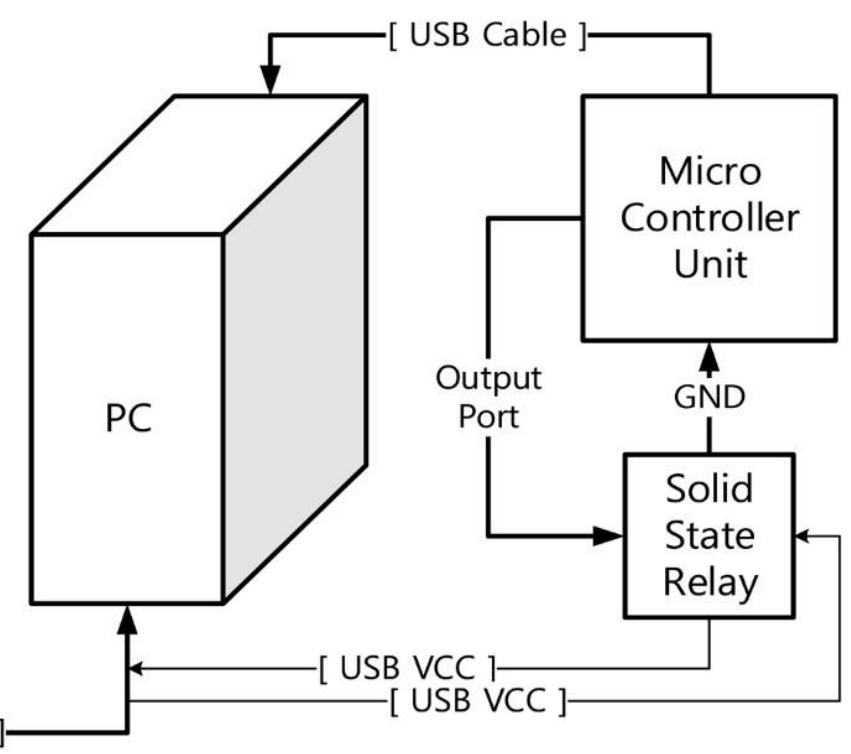

Figure 6. Diagram on SSR and MCU Link of USB Webcam

\subsection{Solid-State Relay (SSR)}

A solid-state relay (SSR) is an electronic switch that, unlike an electromechanical relay, does not have moving parts. It has two responsive types: photosensitive and voltage-responsive. We use the voltage-responsive SSR in this paper [6].

\subsection{Diagram of SSR and MCU Link of USB Webcam}

Once the SSR and MCU are used for a USB device, there is no need for a user to 
physically perform link control, because instantaneous automatic link control through programming is available when needed.

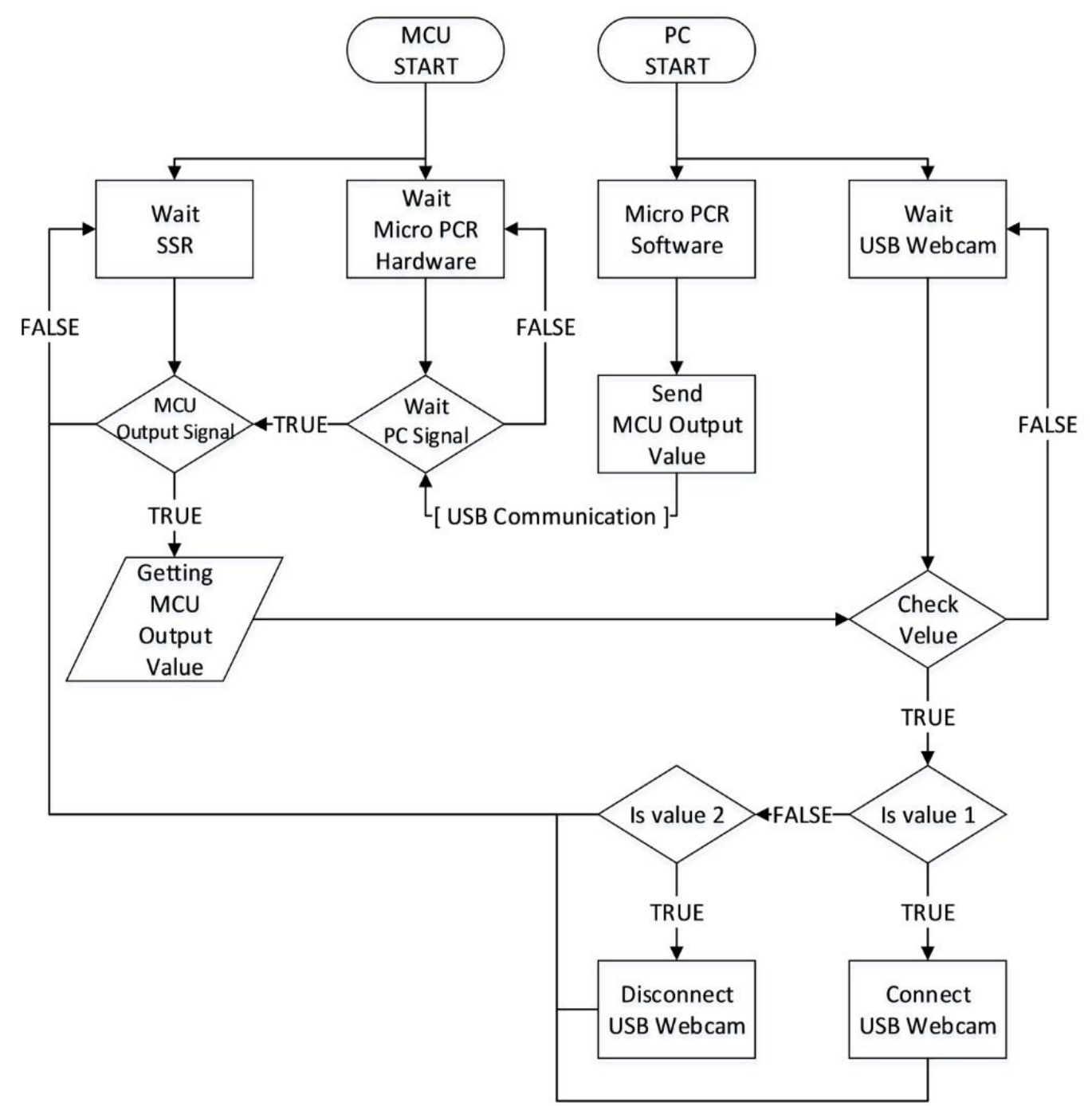

Figure 7. USB Camera Operation Flow Diagram Using MCU and SSR Control

Figure 6 shows a block diagram in which a USB camera is connected to the SSR and MCU. As in Figure 3, the USB VCC jumper wires are disconnected and the ends of the jumper wires are connected to each pin that is suitable for the SSR. An SSR cannot be used alone; it needs a medium that can send voltage signals in required situations like an MCU. In this study, the medium that sends voltage signals is an MCU that is used in a micro PCR. The output and ground ports of the MCU are linked to each pin of the SSR. The USB camera to which both the SSR and MCU are connected controls its VCC, using the MCU output signal, and will be turned on and off by a program as needed.

\subsection{USB Camera Operation Diagram}

Figure 7 shows the flow diagram in which the MCU is operated by the command from the PC program and the MCU controls the SSR to control the USB camera. Once the program sends a command to the MCU, the MCU sends a voltage signal to the SRR. Upon receiving the signal, the SSR, as well as the VCC of the USB camera, switches on, enabling the PC to recognize the USB camera. When the SSR receives a voltage signal from the MCU again, however, it switches off. Similarly, the VCC of the USB camera 
switches off and the PC recognizes that the USB camera is disconnected.

\section{Discussion and Results}

The following describes how we actually operate a micro PCR. First, we execute the PC software that operates the micro PCR.

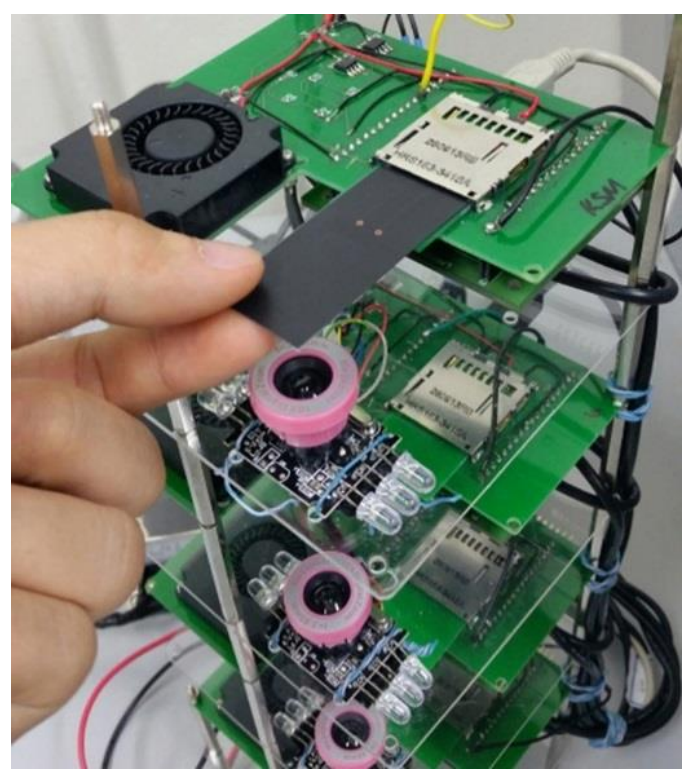

Figure 8. Mounted Biochip

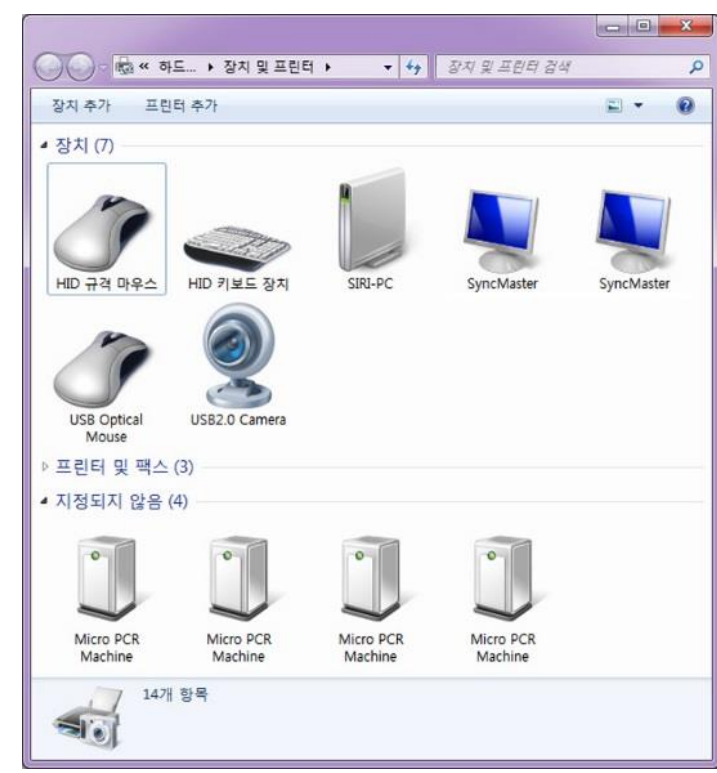

Figure 9. PC and USB Webcam Connection

As shown in Figure 8, the software recognizes when a biochip is mounted on a micro PCR. When the micro PCR recognizes the QR code attached to the bottom of the biochip, it sends a command via software to the MCU to activate the SSR. When the SSR starts to operate, the USB webcam installed on the micro PCR also starts to operate, and as shown in Figure 9, the PC can also recognize the USB web cameras.

When the USB webcam is operated, the software shows a preview window to recognize the QR code, as shown in Figure 10, and once the QR code is recognized, the preview window is closed. The chip temperature correction factor value from the $\mathrm{QR}$ code is calculated with the temperature value obtained from the biochip temperature sensor, which is mounted on the micro PCR. Figure 11 shows the software that operates a micro PCR, the chip temperature correction factor value that was obtained from the corrected temperature of a biochip, and the QR code. 


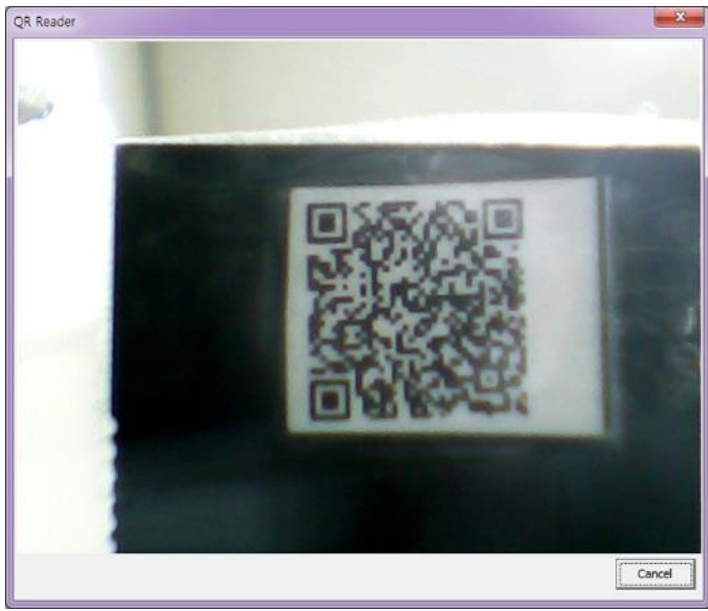

Figure 10. Webcam Preview Window

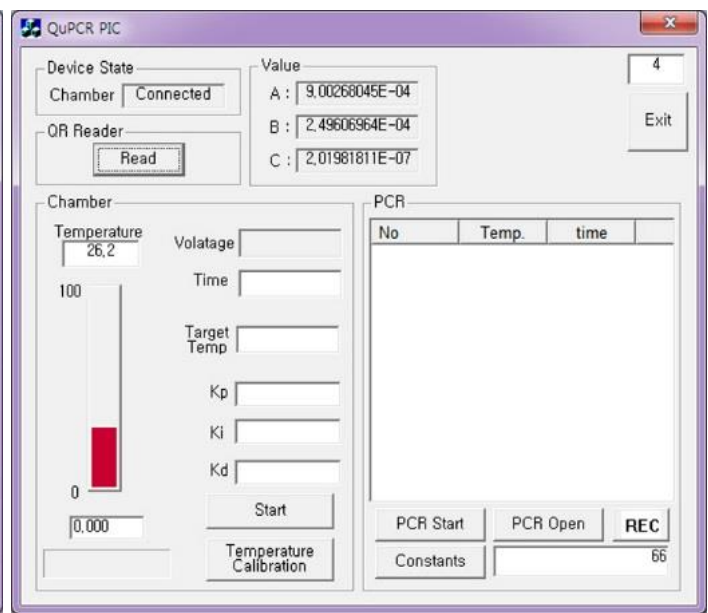

Figure 11. QR Code Data Output

\section{Conclusion}

The on/off control of the USB cameras is needed to compute the QR code as a biochip correction factor value of the micro PCR and to recognize the QR code. Generally, a user controls the connection of a USB device in person because a PC can control only one camera. However, attaching and detaching connectors to the USB ports of a PC in person will be difficult and inconvenient, as well as time-consuming, as the number of devices that need controlling increases. Thus, this paper proposed a USB device control system using MCU and SSR. Using this system, time for preparation and tasks reduces, and work comfort increases. In addition, we can use the proposed system if USB devices other than the USB camera need to be connection controlled.

\section{Acknowledgments}

The research was supported by Basic Science Research Program through the National Research Foundation of Korea (NRF) funded by the Ministry of Education, Science and Technology (2012R1A1A2040381).

\section{References}

[1] B. H. Park, J. H. Jung, Y. T. Kim, T. S. Seo, "Development of a Fully Integrated Genetic Analysis Microsystem", Physics \& High Technology, vol. 3, no. 9, (2013).

[2] C. Zhang and D. Xing, "Miniaturized PCR chips for nucleic acid amplification and analysis: latest advances and future trends", Nucleic Acids Res., vol. 35, no. 13, (2007).

[3] C.-Y. Park, J.-D. Kim, J.-H. Ku, Y.-S. Kim, H.-J. Song and J. Kim, "Printed Circuit Board-Based Polymerase Chain Reaction Chip", Sensor Letters, vol. 10, no. 5/6, (2012)

[4] http://www.qrcode.com/en/

[5] http://www.usb.org/developers/docs/usb20_docs

[6] http://en.wikipedia.org/wiki/Solid-state_relay

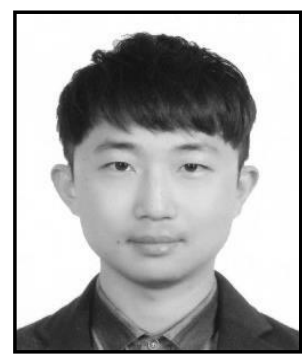

\section{Authors}

Seung-Cheol Lee. He is enrolled in a fourth-year student in Computer Engineering major in Hallym University. He currently studies for a master's degree in Computer Engineering at Hallym University. His research interests are in Bio-IT convergence, embedded system. 

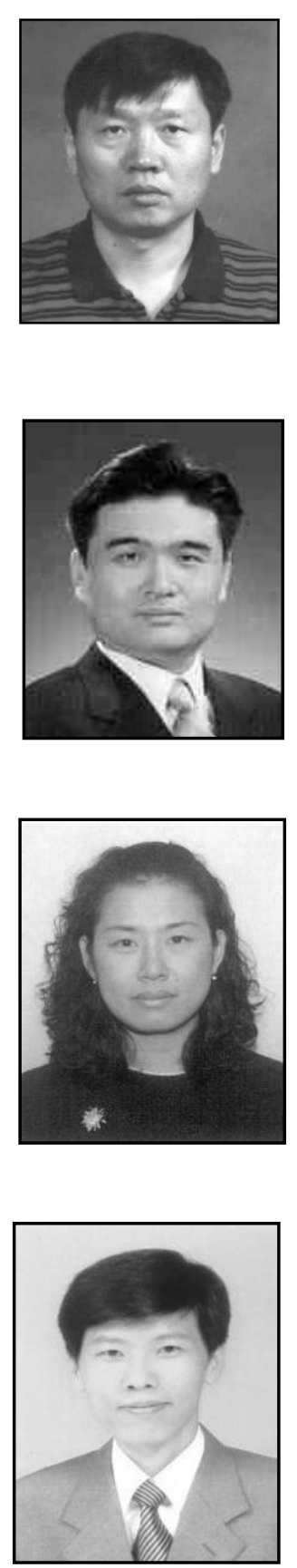

Jong-Dae Kim. He received the M.S. and the Ph.D. degrees in Electrical Engineering from Korea Advanced Institute of Science and Technology, Seoul, Korea, in 1984 and 1990, respectively. He worked for Samsung Electronics from 1988 to 2000 as an electrical engineer. $\mathrm{He}$ is a Professor in Department of Ubiquitous Computing, Hallym University. His recent interests focus on biomedical system and bioinformatics.

Yu-Seop Kim. He received the Ph.D. degree in Computer Engineering from Seoul National University. He is currently a Professor in the Department of Ubiquitous Computing at Hallym University, South Korea. His research interests are in the areas of bioinformatics, computational intelligence and natural language processing.

Hye-Jeong Song. She received the Ph.D. degree in Computer Engineering from Hallym University. She is a Professor in Department of Ubiquitous Computing, Hallym University. Her recent interests focuses on biomedical system and bioinformatics

Chan-Young Park. He received the B.S. from Seoul National University and the M.S. and the Ph.D. degree from Korea Advanced Institute of Science and Technology in 1989 and 1995, respectively. From 1991 to 1999, he worked at Samsung Electronics. He is currently a Professor in the Department of Ubiquitous Computing of Hallym University, Korea. His research interests are in Bio-IT convergence, Intelligent Transportation System and sensor networks 